\title{
Perioperative Pregabalin for Postoperative Pain Relief after Thoracotomy
}

\author{
Tatiana Sidiropoulou ${ }^{1 *}$, Evangelos Giavasopoulos ${ }^{2}$, Georgia Kostopanagiotou ${ }^{1}$, Marianthi Vafeiadou ${ }^{2}$, \\ Achilleas Lioulias $^{3}$, Emmanouil Stamatakis $^{1}$, Paraskevi Matsota ${ }^{1}$
}

${ }^{1}$ Second Department of Anaesthesiology, University of Athens, Attikon Hospital, Athens, Greece

${ }^{2}$ Department of Anaesthesiology, Sismanoglion Hospital, Athens, Greece

${ }^{3}$ Department of Thoracic Surgery, Sismanoglion Hospital, Athens, Greece

"Corresponding author: Tatiana Sidiropoulou, Second Department of Anaesthesiology, University of Athens, Attikon Hospital, Rimini 1, Haidari, 12462, Athens, Greece, E-mail: tatianasid@gmail.com

\begin{abstract}
Background: Pregabalin is effective both at controlling postoperative pain and preventing chronic neuropathic pain. Local anaesthetic infiltration is a simple and inexpensive method to provide postoperative analgesia. In this study we tested the hypothesis that pregabalin administered perioperatively combined with continuous wound infusion will aid better the post-thoracotomy pain management compared to pregabalin monotherapy.

Methods: Forty five patients received either placebo (PLCB), pregabalin (PRG) or pregabalin and continuous wound infusion (PRG $+\mathrm{CWI})$ of local anaesthetics. Postoperative data collection included opioid consumption, VAS scores at rest and during cough. At 1 and 3 months from surgery patients were assessed with the DN4 questionnaire for neuropathic pain.

Results: VAS scores were significantly lower in the PRG+CWI group $(p<0.05)$ at rest while during cough the placebo group had higher scores than both treatment groups $(\mathrm{p}<0.001$ or $\mathrm{p}<0.05)$. Morphine consumption measured at $48 \mathrm{hr}$ PO revealed a significant difference in total morphine; PLCB: $49 \pm 11 \mathrm{mg}, \mathrm{PRG}$ : $33 \pm 10 \mathrm{mg}$ and PRG+CWI: $28 \pm 11 \mathrm{mg}$ ( $<0.001$ between placebo and the other two groups). The incidence of neuropathic pain was more frequent in the placebo group (1-month: PLCB: 10 pt, PRG: 0 pt and PRG+ CWI: 0 pt $(p<0.001), 3$ months: PLCB: 10 pts, PRG: 0 pt and PRG+CWI: 0 pt $(\mathrm{p}<0.001))$.

Conclusions: Perioperative administration of pregabalin significantly reduces pain scores, opioid consumption and incidence of neuropathic pain in post-thoracotomy patients. The addition of continuous wound infiltration of local anaesthetics although improved immediate postoperative analgesia at rest did not affect differently the development of neuropathic pain.
\end{abstract}

Received date: February 3, 2016

Accepted date: February 26, 2016

Published date: February 29, 2016

Citation: Sidiropoulou, T., et al. Perioperative Pregabalin for Postoperative Pain Relief after Thoracotomy. (2016) J Anesth Surg 3(1): 106- 111.

DOI: $10.15436 / 2377-1364.16 .033$

Keywords: Pain postoperative; Analgesics pregabalin; Analgesics morphine;

Thoracotomy; Anesthetics local

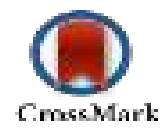

\section{Introduction}

Thoracotomy is one of the surgical procedures associated with most severe acute postoperative pain and it has a high incidence of eliciting persistent post-surgical pain, often lasting months or years ${ }^{[1,2]}$. Pain is not localized only in the site of surgery but can be experienced anywhere in the hemithorax involved ${ }^{[3]}$. More than half of the cases $(35-80 \%)$ comprise a neuropathic component ${ }^{[4,5]}$ including allodynia, dysaesthesia, and burning. It has been demonstrated that the development of chronic pain is associated with duration and severity of immediate postoperative pain and extent of surgery. Effective block of neural afferents reduces acute post-thoracotomy pain and may thereby blunt the development of pain memory ${ }^{[6]}$.

Gabapentinoids (pregabalin and gabapentin) have been effectively used to treat acute postoperative pain and reduce the incidence of chronic post-surgical pain ${ }^{[7,8]}$. Gabapentin has not been of analgesic benefit in the particular setting of post-thoracoto-

Copyrights: (C) 2016 Sidiropoulou, T. This is an Open access article distributed under the terms of Creative Commons Attribution 4.0 International License. 
my patients as a single preoperative dose ${ }^{[9,10]}$ or if administered pre and postoperatively ${ }^{[11]}$. Pregabalin has a more reliable pharmacokinetic profile ( $\geq 90 \%$ bioavailability of a single dose) and may have a more promising and effective role in the prevention of chronic post-surgical pain ${ }^{[12,13]}$ while being safer in the elder$1 y^{[12]}$. However in the thoracic surgery setting, data regarding its use are limited and conflicting ${ }^{[14,15]}$

Central blocks are the common practice for thoracic surgery perioperative management, in the absence of any contraindication. As an alternative, would infiltrations have shown to be an effective technique for management of surgical pain, since they were correlated with less pain in different clinical settings ${ }^{[16,17]}$. In addition to providing good analgesia, local anaesthetic wound infiltration is simple, safe and inexpensive. A systematic review found that continuous wound instillation was effective in reducing pain scores and had opioid-sparing effects for most surgical subgroups ${ }^{[18]}$. However, the benefit of this technique remains controversial after thoracic surgery ${ }^{[19-21]}$, mainly because of its limited use. Thus, taking into consideration that many thoracotomies are performed under general anesthesia alone, it is deemed that an optimal pain management method should be addressed for those patients.

In this study we aimed to determine the efficacy of perioperative pregabalin, with or without the addition of a local anaesthetic continuous wound infusion, in the control of postoperative pain and the prevention of chronic post-thoracotomy pain in patients undergoing thoracotomy under general anesthesia alone.

\section{Methods}

This randomised controlled double-blind study obtained approval from the Institutional Ethics Committee and Scientific Council of the Attikon Hospital and Sismanoglion Hospital (protocol No 318/15.09.10 and 0591/08.02.08 respectively). The trial was registered in clinicaltrials.gov (registration number: NCT01726205). During the time period between October 2008 and May 2012, 63 eligible patients undergoing elective thoracotomy under general anaesthesia alone were identified. Twelve patients refused to participate, while six were excluded for logistical reasons (emergency surgery, placement of epidural catheter, other) (see Figure. 1). Therefore 45 patients were consecutively enrolled in the study after written informed consent was obtained. Subsequently they were randomly allocated (with a computer generated list of random numbers) in one of three groups and received placebo drug and saline infusion (group PLCB), pregabalin and saline wound infusion (group PRG), or pregabalin and ropivacaine wound infusion $(\mathrm{PRG}+\mathrm{CWI})$.

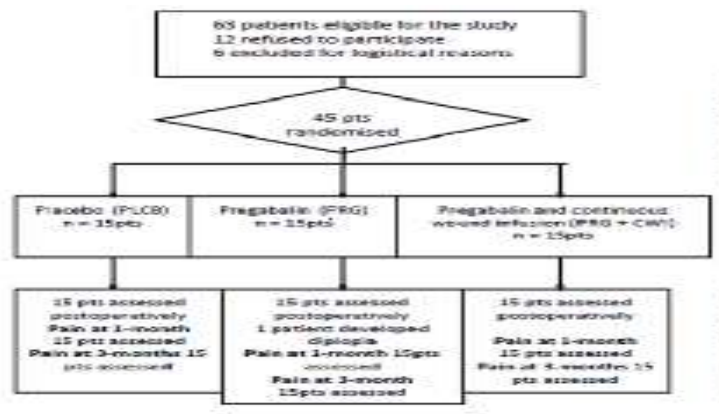

Figure 1: Flow Diagram of the study.
Exclusion criteria from the study were age $>70$ years, $\mathrm{BMI}>30$, pre existent chronic pain, neurologic disease, alcohol or opioid abuse, pre existing treatment with analgesics, anxiolytics, sedatives, antidepressants, calcium channel inhibitors, or any contraindication for the use of patient controlled analgesia (PCA). The placebo drug was manufactured identical in size and colour to pregabalin in our hospital pharmacy. A hospital nurse, in charge of the study randomisation administered the medication to the patients. The medication (drug or placebo) was packed in sealed envelopes where only the patient number was visible and the name of the study. The same person prepared the postoperative wound infusions (normal saline or local anaesthetic) and was not otherwise involved in the study. Patients were advised to take pregabalin $75 \mathrm{mg}$ (group PRG and PRG+CWI) or placebo (group PLCB) every 12 hours, starting the afternoon before surgery $(8.00 \mathrm{pm})$ and continuing for the first 5 postoperative days (POD\#5). At the same time, the PCA device and Visual Analogue Scale (0-10) were shown and explained to all patients, in order to familiarize themselves with the usage of device and method of assessment, respectively.

Patients were not premedicated. Intraoperatively, anaesthesia was induced with propofol (2 - $2.5 \mathrm{mg} / \mathrm{kg})$ and fentanyl $(5 \mu \mathrm{g} / \mathrm{kg})$ and tracheal intubation was facilitated with cis-atracurium $(0.15-0.20 \mathrm{mg} / \mathrm{kg})$. Anaesthesia was maintained with sevoflurane in an $\mathrm{O}_{2}$ /air mixture. All patients received the same anaesthetic drugs; however modifications in drug dosage were at the discretion of the anaesthesist in charge. A left double lumen tube was placed in all patients. Standard monitoring with invasive blood pressure measurement was applied in all patients. To monitor the depth of anaesthesia, a Bispectral Index (BIS) sensor was attached to the patient's forehead and connected to a BIS monitor (BIS A-2000, Aspect Medical Systems Inc., USA). Thirty minutes before the end of surgery all patients received ondasetron $4 \mathrm{mg}$ and paracetamol $1 \mathrm{~g}$ intravenously. Before wound closure the surgeon placed a 20-gauge multi-orifice catheter (PAIN fusor Catheter Baxter, France) just above the sutured lattisimus dorsi muscle layer. The catheter had a distribution length for local anaesthetics of $15 \mathrm{~cm}$. The catheter was attached to the postoperative infusion via an elastomeric pump (SV2 Infusor, Baxter, France) which distributed either normal saline (PLCB or $\mathrm{PRG}$ group) or ropivacaine $0.75 \%$ ( $\mathrm{PRG}+\mathrm{CWI}$ group) for the first 48 hours postoperatively with a flow rate of $5 \mathrm{ml} / \mathrm{hr}$. After this period the catheter was removed aseptically. For the first 48 postoperative hours all patients received patient-controlled analgesia via a PCA device (RythmicTM Plus - Medical Devices, Greece) with morphine $1 \mathrm{mg} / \mathrm{mL}$ set at boluses of $1 \mathrm{mg}$ each with a lockout period of $7 \mathrm{~min}$ was granted in all patients and systematic administration of paracetamol $1 \mathrm{~g}$ intravenously every 6 hours. Maximum morphine consumption at $4 \mathrm{~h}$ was set at $16 \mathrm{mg}$. In the third postoperative day (POD\#3) the PCA device and intravenous paracetamol were discontinued and patients were transitioned to oral Lonalgal ${ }^{\circledR}$ tablets (paracetamol $500 \mathrm{mg}$ and codeine $30 \mathrm{mg}$ ) (Boehringer Ingelheim, Germany) until POD\#5. The tablets were given as needed for adequate pain control every time the patient complained of pain (VAS $\geq 3 \mathrm{~cm}$ ) up to a maximum of 4 tablets daily.

Pain levels were assessed at rest and during cough with a visual analogue scale $(0-100 \mathrm{~mm})$ together with potential side-effects such as sedation, nausea, vomiting, dizziness, postoperative headache, stipsis, urinary retention, vertigo, and 
blurred vision. Data were collected at 2, 4, 8 hours postoperatively and then each day from POD\#1 to POD\#5. Morphine consumption was collected via the device's internal memory at the same time periods as VAS scores. Sedation levels were documented by using an in-house score ( 0 , awake; 1 , sleeping, but easily arousable; 2 , sleeping, hardly arousable; 3 , not arousable). At POD\#5 a patient satisfaction score for the analgesic efficacy was also collected (1-insufficient, 2-sufficient, 3-good, 4-very good). Subsequently patients were interviewed at one and three months after surgery in a regular surgical visit regarding the presence of persistent pain, the development of neuropathic pain and analgesic consumption. These time points mark the transition from acute, late pain to chronic pain. Investigators used a 4 point verbal score for pain (0-no pain to 3 -severe pain) and the Douleur Neuropathique 4 (DN4) questionnaire, to determine the presence of neuropathic pain in the operated side ${ }^{[22]}$. The DN4 questionnaire has been translated and validated in the greek language $^{[23]}$. It consists of 4 questions (10 items) regarding neuropathic pain with a maximum score of 10. A DN4 score of 4 or more is positive for the presence of neuropathic pain. Patients who had persistent post-thoracotomy pain 3 months after surgery were referred to our ambulatory Pain Service for follow up. The hypothesis tested in this study was that, in patients undergoing thoracotomy under general anesthesia alone, the addition of continuous wound infusion to pregabalin administered perioperatively will aid better the post-thoracotomy pain management compared to pregabalin monotherapy in terms of the efficacy of provided postoperative analgesia and the prevention of chronic post-thoracotomy pain. Sample size calculation was based on the null hypothesis of no pain difference across all groups. Assuming a baseline pain intensity of 5 on a $0-100 \mathrm{~mm}$ VAS scale and a standard deviation of 2 we calculated that a sample of 14 patients per group would provide an $80 \%$ chance of detecting a mean difference of 2 among treatment groups (our primary endpoint). To account for three treatment groups we used the Bonferroni inequality to calculate sample size (where the level of significance is divided by the number of groups $\alpha / 3=0.0167$ ). We enrolled 15 patients per group to allow for dropouts. Secondary endpoints included opioid consumption, sedation rate, incidence of side effects, patient satisfaction, and persistance of post-thoracotomy pain and neuropathic pain at 1 and 3 months postoperatively (verbal rating score, DN4).

Demographic, intraoperative and postoperative continuous data were analyzed with the Kruscal-Wallis test for k samples (a non parametric analysis of variance for data measured at one single point). If a significant result was found we performed pairwise comparisons with the Mann Whitney-U test. Nominal and ordinal data were analyzed using the Pearson's chi square test and confirmed with exact probability tests because of the small sample size. Repeated measures such as pain, morphine consumption, oral analgesics and sedation scores were analysed using a linear mixed model with treatment, time and their interaction as fixed effects. If a significant result was obtained then the treatment groups were compared at each time point in pair wise comparisons. The Bonferroni correction was applied for multiple comparisons. Descriptive statistics are reported as mean and SD for continuous variables, median (range) for ordinal variables, and count and/or percentages (\%) for dichotomous variables. Statistics were performed using the SPSS v17.0 (SPSS Inc, Chicago, USA).

\section{Results}

Forty-five patients completed the study (Figure 1). One patient from the pregabalin (PRG) group developed severe but transient diplopia during the second postoperative day and therefore pregabalin administration was discontinued. However this patient continued to receive opioid analgesia and postoperative data were collected and included in the analysis (intention to treat).

There were no significant differences in patient demographic and intra operative data (Table 1). A significant effect of time and treatment was found in visual analogue scale (VAS) scores at rest (time: $p<0.001$, treatment: $p=0.015$ ) and after cough (time: $p<0.001$, treatment: $p<0.001$ ) but their interaction (treatment*time) was not significant in any instance. Subsequent pair wise analysis revealed that VAS scores were significantly lower in the $\mathrm{PRG}+\mathrm{CWI}$ group at rest while during cough the placebo group had higher scores than both treatment groups (Table 2). The same model used for morphine consumption resulted in a significant effect of time and treatment $(\mathrm{p}<0.001$ and $\mathrm{p}<$ 0.001 respectively) but not their interaction (Figure 2). Cumulative morphine consumption measured at $48 \mathrm{hr}$ postoperatively revealed a significant difference in total morphine between the three groups; PLCB: $49.2 \pm 11.4 \mathrm{mg}$, PRG: $32.9 \pm 10 \mathrm{mg}$ and PRG+CWI: $28.5 \pm 10.8 \mathrm{mg}(\mathrm{p}<0.001$ between placebo and the other two groups). Lonalgal ${ }^{\circledR}$ consumption (codeine and paracetamol) analysis revealed a significant effect of treatment $(p<$ $0.001)$. The pregabalin group was administered less codeine and paracetamol tablets than the other two groups during the $3^{\text {rd }}(\mathrm{p}=$ $0.004), 4^{\text {th }}(\mathrm{p}=0.044)$ and $5^{\text {th }}(\mathrm{p}=0.010)$ postoperative day.

Table 1: Demographic and intraoperative data.

\begin{tabular}{|l|l|l|l|l|}
\hline & PLCB & PRG & PRG+CWI & $p$ \\
\hline Age (ys) & $59 \pm 14$ & $57 \pm 7$ & $56 \pm 14$ & $0.778 \dagger$ \\
\hline $\begin{array}{l}\text { Body Mass Index } \\
\left(\mathrm{kg} / \mathrm{m}^{2}\right)\end{array}$ & $25.4 \pm 3.0$ & $24.8 \pm 2.8$ & $23.3 \pm 3.2$ & $0.155 \dagger$ \\
\hline Sex (M/F) & $11 / 4$ & $13 / 2$ & $12 / 3$ & $0.655 \dagger$ \\
\hline ASA (I/II/III) & $1 / 12 / 2$ & $4 / 9 / 2$ & $2 / 10 / 3$ & 0.603 \\
\hline $\begin{array}{l}\text { Thoracotomy } \\
\text { (Right/Left) }\end{array}$ & $12 / 3$ & $10 / 5$ & $9 / 6$ & $0.484 \dagger$ \\
\hline Type of Surgery & \multicolumn{5}{|l}{} \\
\hline Pneumonectomy & 0 & 4 & 4 & \\
\hline Lobectomy & 10 & 10 & 9 & $0.106 \ddagger$ \\
\hline Wedge Resection & 3 & 0 & 0 & \\
\hline Open pleurectomy & 2 & 2 & 1 & $0.713 \dagger$ \\
\hline $\begin{array}{l}\text { Duration of surgery } \\
\text { (min) }\end{array}$ & $148 \pm 24$ & $157 \pm 45$ & $156 \pm 31$ & \\
\hline
\end{tabular}

PLCB: placebo group; PRG: pregabalin group, $\mathrm{PRG}+\mathrm{CWI}$ : pregabalin and continuous wound infusion group

$\dagger$ : analysis of Variance,

t: chi-square test 
Table 2: Visual Analogue Scale scores. Numbers represent mean (95\% confidence intervals)

\begin{tabular}{|c|c|c|c|}
\hline & PLCB & PRG & PRG+CWI \\
\hline \multicolumn{4}{|l|}{ VAS $2 \mathrm{~h}$} \\
\hline at rest & $42(36-48)$ & $36(31-43)$ & $29(25-32)^{*}$ \\
\hline $\begin{array}{l}\text { during } \\
\text { cough }\end{array}$ & $70(67-73)$ & $62(57-67)^{* *}$ & $48(43-52)^{*}$ \\
\hline \multicolumn{4}{|l|}{ VAS 4h } \\
\hline at rest & $40(35-45)$ & $33(27-39)$ & $27(23-30)^{*}$ \\
\hline $\begin{array}{l}\text { during } \\
\text { cough }\end{array}$ & $67(63-71)$ & $55(50-59)^{*}$ & $50(45-57)^{*}$ \\
\hline \multicolumn{4}{|l|}{ VAS 8h } \\
\hline at rest & $39(35-44)$ & $33(28-39)$ & $25(25-28)^{*}$ \\
\hline $\begin{array}{l}\text { during } \\
\text { cough }\end{array}$ & $65(61-69)$ & $53(49-56)^{*}$ & $51(48-55)^{*}$ \\
\hline \multicolumn{4}{|c|}{ VAS 24h } \\
\hline at rest & $39(34-45)$ & $31(26-37)$ & $26(23-30)^{*}$ \\
\hline $\begin{array}{l}\text { during } \\
\text { cough }\end{array}$ & $67(62-72)$ & $52(46-58)^{*}$ & $54(48-60)^{* *}$ \\
\hline \multicolumn{4}{|l|}{ VAS 2d } \\
\hline at rest & $41(35-46)$ & $31(23-39)$ & $27(20-35)^{* *}$ \\
\hline $\begin{array}{l}\text { during } \\
\text { cough }\end{array}$ & $67(63-72)$ & $51(46-56)^{*}$ & $55(47-62)^{* *}$ \\
\hline \multicolumn{4}{|l|}{ VAS 3d } \\
\hline at rest & $36(31-41)$ & $27(20-34)$ & $24(18-31)^{* *}$ \\
\hline $\begin{array}{l}\text { during } \\
\text { cough }\end{array}$ & $57(53-62)$ & $43(37-48)^{*}$ & $48(42-55)$ \\
\hline \multicolumn{4}{|l|}{ VAS 4d } \\
\hline at rest & $33(29-38)$ & $26(19-33)$ & $21(14-27)^{* *}$ \\
\hline $\begin{array}{l}\text { during } \\
\text { cough }\end{array}$ & $56(53-59)$ & $41(35-46)^{*}$ & $43(37-50)^{* *}$ \\
\hline \multicolumn{4}{|l|}{ VAS 5d } \\
\hline at rest & $31(28-35)$ & $25(18-31)$ & $20(14-26)^{* *}$ \\
\hline $\begin{array}{l}\text { during } \\
\text { cough }\end{array}$ & $51(47-55)$ & $39(34-44)^{*}$ & $40(35-45)^{* *}$ \\
\hline
\end{tabular}

PLCB: placebo group; PRG: pregabalin group, PRG + CWI: pregabalin and continuous wound infusion group. ${ }^{*} \mathrm{p}<0.001,{ }^{* *} \mathrm{p}<0.05$ vs. the placebo group.

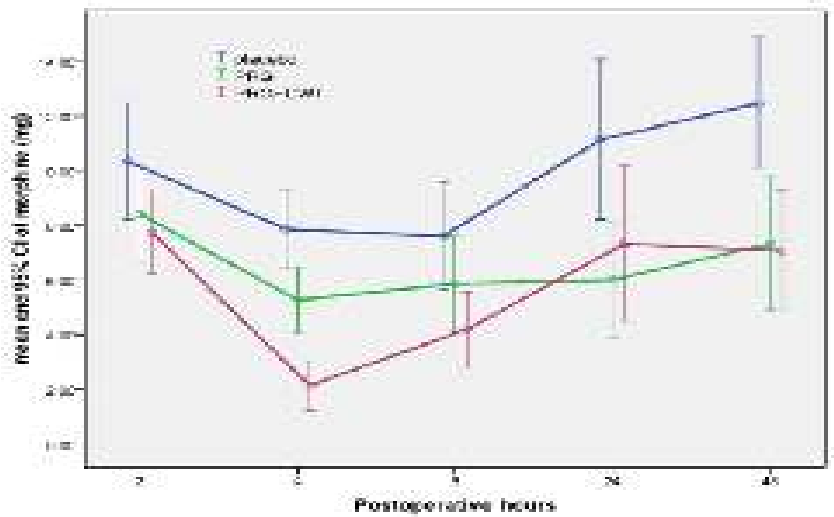

Figure 2: Morphine consumption (mg) at 2, 4, 8, 24 and $48 \mathrm{~h}$ postoperatively in the placebo and treatment groups. 2-hour: no difference between groups. 4-hour: PLCB $p<0.01$ vs. other groups, 8-hour: PLCB vs. $P R G+C W I p<0.05$, 24-hour: PLCB vs. PRG $p<0.05$ and 48-hour: PLCB $\mathrm{p}<0.001$ vs. other groups.
Incidence of side effects was similar among groups with the exception of nausea and vomit us which were lower in the treatment groups (Table 3). No differences were observed in sedation scores throughout the study period. Patients in the placebo group were less satisfied than patients in the treatment groups (Table 3).

Table 3: Incidence of side effects and patient satisfaction.

\begin{tabular}{|l|l|l|l|l|}
\hline & PLCB & PRG & PRG+CWI & $\boldsymbol{p}^{*}$ \\
\hline Nausea & $11(73 \%)$ & $6(40 \%)$ & $0(0 \%)$ & $<0.001$ \\
\hline Vomitus & $4(27 \%)$ & $0(0 \%)$ & $0(0 \%)$ & 0.027 \\
\hline Pruritus & & $0(0 \%)$ & $0(0 \%)$ & 1 \\
\hline Constipation & $3(20 \%)$ & $3(20 \%)$ & $0(0 \%)$ & 0.33 \\
\hline Urinary Retention & $1(7 \%)$ & $0(0 \%)$ & $0(0 \%)$ & 1 \\
\hline Vertigo & $12(80 \%)$ & $13(87 \%)$ & $8(57)$ & 0.191 \\
\hline Headache & $0(0 \%)$ & $0(0 \%)$ & $2(14 \%)$ & 0.096 \\
\hline Confusion & $0(0 \%)$ & $0(0 \%)$ & $1(7 \%)$ & 0.318 \\
\hline Blurred vision & $0(0 \%)$ & $0(0 \%)$ & $1(7 \%)$ & 0.318 \\
\hline Patient Satisfaction & 11 & 1 & 0 & \\
\hline insufficient & 4 & 0 & 0 & $<0.001$ \\
\hline sufficient & 0 & 8 & 9 & \\
\hline good & 0 & 6 & 6 & \\
\hline excellent & & & & \\
\hline
\end{tabular}

Numbers represent incidence of effect (percentage within group).

PLCB: placebo group; PRG: pregabalin group, PRG + CWI: pregabalin and continuous wound infusion group.

* Two-sided exact significance test.

The presence of pain at 1- and 3-months postoperatively was more frequent in the placebo group than the PRG or the PRG+CWI group. At 1-month all patients in the placebo group complained of light $(n=4)$ or moderate $(n=11)$ pain whereas in the PRG group 2 patients complained of light and 2 of moderate pain. In the $\mathrm{PRG}+\mathrm{CWI}$ group 3 patients complained of light pain and one of moderate pain $(\mathrm{p}<0.001)$. At the 3 month interview 7 patients in the placebo group complained of light and 5 of moderate pain while only one patient in the PRG group complained of light pain and none in the PRG + CWI group $(\mathrm{p}<0.001)$.

Accordingly, incidence of neuropathic pain (DN4 > 4) was more frequent in the placebo group (placebo: $10(66 \%)$ patients versus none $(0 \%)$ in the PRG group and the PRG + CWI group $(\mathrm{p}<0.001)$. At 3 -months $10(66 \%)$ patients in the placebo group complained of symptoms of neuropathic pain versus none $(0 \%)$ in the PRG and PRG + CWI group $(p<0.001)$. There was a significant correlation between verbal pain and DN4 scores ( $p$ $<0.001)$.

\section{Discussion}

In this study we found a significant effect of perioperative pregabalin administration in reducing pain scores and opioid consumption after thoracotomy. Accordingly nausea and vomiting were decreased in the pregabalin groups. In addition patient satisfaction was higher in the treatment groups. Pregabalin was also effective in preventing long term pain development and the 
development of neuropathic pain, hyperalgesia and allodynia as measured at 1 and 3 months postoperatively with the DN4 score. The addition of continuous wound infiltration although improved postoperative analgesia (lower VAS scores both at rest and during cough compared to placebo group) in the immediate postoperative period did not provide superior pain control with respect to the incidence of persistent post-thoracotomy pain and the development of neuropathic pain.

In the present study persistent post thoracotomy pain was assessed using the verbal rating scale. In the pregabalin groups a minimal percentage of patients referred light or moderate pain at 1 month that was not present at 3 months postoperatively while no patient developed neuropathic pain. Conversely in the placebo group $67 \%$ pts presented persistent pain at 1 month and neuropathic pain at 3 months. The presence of pain and neuropathic pain correlated in our study. This is in accordance with previous data indicating that while a neuropathic component is not always present in patients suffering from chronic post-thoracotomy pain ${ }^{[24,25]}$, more severe pain is associated with neuropathic pain and the consumption of oral opioid analgesics.

Recently, two published studies have also investigated the use of pregabalin in thoracotomy pts and yielded contradictory results ${ }^{[14,15]}$. Both studies included an epidural technique for anesthesia and postoperative analgesia. In the study by Matsutani et al a similar dosage with ours of pregabalin was used but was continued for a longer postoperative period (15 days $)^{[15]}$. Similar to our results this study has documented better postoperative analgesia in the pregabalin group and no component of neuropathic pain in the pregabalin group at 2 months postoperatively. However, the reported incidence of neuropathic pain in the non pregabalin group was lower comparing to our results $(26 \%$ vs. $67 \%)$, a difference which might be owed to the protective effect of epidural anaesthesia. Brulotte et al, did not document a protective effect of gabapentin in both immediate and long term pain management, although they used a doubled daily dose $(300 \mathrm{mg}$ ) with respect to the one used in our study. However they reported a similar percentage of patients $(66 \%)$ with signs and symptoms suggestive of neuropathic pain, which is in accordance with our findings ${ }^{[14]}$.

A distinct, clear effect of continuous infusion of local anaesthetics was not evident from this study. Except from pain at rest, where $\mathrm{PRG}+\mathrm{CWI}$ group achieved lower scores, pain scores at cough, opioid consumption and incidence of post thoracotomy neuropathic pain were comparable between the two treatment groups. There is paucity of randomised controlled trials regarding local anaesthetic wound infiltration in patients undergoing thoracotomy. A study which involved retrospective analysis of 110 patients revealed lower pain scores in the wound infiltration group compared with a continuous thoracic epidural infusion ${ }^{[20]}$. Nevertheless not all patients in the epidural group received the same amount of local anaesthetic or opioid via their epidural catheter, making the comparison questionable. In another study of post thoracotomy patients, lower pain scores and narcotic use were registered in the continuous wound infiltration group when compared to a historical group ${ }^{[19]}$. Conversely in patients undergoing cardiac surgery a continuous bupivacaine infiltration in the sternotomy site did not improve postoperative pain control $^{[21]}$. In this study we failed to observe an additional analgesic effect of the wound infusion in the pregabalin and continuous infusion group.

Nausea and vomiting was lower as expected in the pregabalin groups. Pregabalin is associated with increased incidence of somnolence and dizziness ${ }^{[26,27]}$. In this study, we did not find a significant difference in sedation, or dizziness in the treatment groups. We documented a severe case of diplopia in the pregabalin group which receded after discontinuation of the drug. Noticeably this patient did not develop chronic neuropathic pain at 1 and 3 month visit. We suggest that pregabalin can be a valid alternative for pain management in thoracotomy patients that receive general anesthesia alone and this is also documented from the higher patient satisfaction scores in the treatment groups.

Although a preventive effect of pregabalin on post-thoracotomy pain and neuropathic pain was evidenced by our study, we should note that this study was powered in the immediate postoperative VAS scores and not on the development of neuropathic pain. Another potential limitation of our study, is the fact that we did not involve a group receiving only wound Infiltration, in order to investigate its impact as a sole treatment on immediate and long term post-thoracotomy pain. Nevertheless pain scores and morphine consumption were similar between the 2 treatment groups.

\section{Conclusion}

In conclusion this study has shown that in patients undergoing thoracic surgery under general anaesthesia alone, the perioperative administration of pregabalin significantly reduces pain scores and opioid consumption in post-thoracotomy patients. Moreover pregabalin reduced the incidence of hyperalgesia, allodynia and the development of neuropathic pain. Continuous wound instillation of local anaesthetics though slightly improved immediate postoperative analgesia at rest failed to demonstrate any additional analgesic benefit regarding the prevention of neuropathic pain in this study population. Further investigation to confirm the results of the present study as well as to elucidate the mechanisms of the analgesic effect of pregabalin in the different aspects of post-thoracotomy pain is needed.

\section{References}

1. Gottschalk, A., Cohen, S.P., Yang, S., et al. Preventing and treating pain after thoracic surgery. (2006) Anesthesiology 104(3): 594-600.

2. Ochroch, E.A., Gottschalk, A., Augoustides, J., et al. Longterm pain and activity during recovery from major thoracotomy using thoracic epidural analgesia. (2002) Anesthesiology 97(5): 1234-1244.

3. Niraj, G., Rowbotham, D.J. Persistent postoperative pain: where are we now? (2011) Br J Anaesth 107(1): 25-29.

4. Steegers, M.A., Snik, D.M., Verhagen, A.F., et al. Only half of the chronic pain after thoracic surgery shows a neuropathic component. (2008) J Pain 9(10): 955-961.

5. Maguire, M.F., Ravenscroft, A., Beggs, D., et al. A questionnaire study investigating the prevalence of the neuropathic component of chronic pain after thoracic surgery. (2006) Eur J Cardiothorac Surg 29(5): 800-805.

6. Pluijms, W.A., Steegers, M.A., Verhagen, A.F., et al. Chronic post-thoracotomy pain: a retrospective study. (2006) Acta An- 
aesthesiol Scand 50(7): 804-808.

7. Clarke, H., Bonin, R.P., Orser, B.A., e al. The Prevention of Chronic Postsurgical Pain Using Gabapentin and Pregabalin: a Combined Systematic Review and Meta-Analysis. (2012) Anesth Analg 115(2): 428-442.

8. Dauri, M., Faria, S., Gatti, A., et al. Gabapentin and pregabalin for the acute post-operative pain management. A systematic-narrative review of the recent clinical evidences. (2009) Curr Drug Targets 10(8): 716-733.

9. Huot, M.P., Chouinard, P., Girard, F., et al. Gabapentin does not reduce post-thoracotomy shoulder pain: a randomized, double-blind placebo-controlled study. (2008) Can J Anaesth 55(6): 337-343.

10. Kinney, M.A., Mantilla, C.B., Carns, P.E., et al. Preoperative gabapentin for acute post-thoracotomy analgesia: a randomized, double-blinded, active placebo-controlled study. (2012) Pain Pract 12(3): 175-183.

11. Grosen, K., Drewes, A.M., Højsgaard, A., et al. Perioperative gabapentin for the prevention of persistent pain after thoracotomy: a randomized controlled trial. (2014) Eur J Cardiothoracic Surg 46(1): 76-85.

12. Zhang, J., Ho, K.Y., Wang, Y. Efficacy of pregabalin in acute postoperative pain: a meta-analysis. (2011) Br J Anaesth 106(4): 454-462.

13. Bornemann-Cimenti, H., Lederer, A.J., Wejbora, M., et al. Preoperative pregabalin administration significantly reduces postoperative opioid consumption and mechanical hyperalgesia after transperitoneal nephrectomy. (2012) Br J Anaesth 108(5): 845-849.

14. Brulotte, V., Ruel, M.M., Lafontaine, E., et al. Impact of pregabalin on the occurrence of post-thoracotomy pain syndrome : a randomized trial. (2015) Reg Anesth Pain Med 40(3): 262-269. 15. Matsutani, N., Dejima, H., Takahashi, Y., et al. Pregabalin reduces post-surgical pain after thoracotomy: a prospective, randomized, controlled trial. (2014) Surg Today 45(11): 1411-1416. 16. Rawal, N., Axelsson, K., Hylander, J., et al. Postoperative patient-controlled local anesthetic administration at home. (1998) Anesth Analg 86(1): 86-89.
17. Fredman, B., Shapiro, A., Zohar, E., et al . The analgesic efficacy of patient-controlled ropivacaine instillation after cesarean delivery. (2000) Anesth Analg 91(6): 1436-1440.

18. Liu, S.S., Richman, J.M., Thirlby, R.C., et al. Efficacy of continuous wound catheters delivering local anesthetic for postoperative analgesia: a quantitative and qualitative systematic review of randomized controlled trials. (2006) J Am Coll Surg 203(6): 914-932.

19. Salvemini, S. New intraoperative therapeutic approach to control post-thoracotomy pain in patients who underwent main resection due to lung neoplasm. (2008) Minerva Chir 63(5): 335-339.

20. Wheatley, G.H., Rosenbaum, D.H., Paul, M.C., et al. Improved pain management outcomes with continuous infusion of a local anesthetic after thoracotomy. (2005) J Thorac Cardiovasc Surg 130(2): 464-468.

21. Magnano, D., Montalbano, R., Lamarra, M., et al. Ineffectiveness of local wound anesthesia to reduce postoperative pain after median sternotomy. (2005) J Card Surg 20(4): 314-318.

22. Bouhassira, D., Attal, N., Alchaar, H., et al. Comparison of pain syndromes associated with nervous or somatic lesions and development of a new neuropathic pain diagnostic questionnaire (DN4). (2005) Pain 114(1-2): 29-36.

23. Van Seventer, R., Vos, C., Meerding, W., et al. Linguistic validation of the DN4 for use in international studies. (2010) Eur J Pain 14(1): 58-63.

24. Steegers, M.A., Snik, D.M., Verhagen, A.F., et al. Only half of the chronic pain after thoracic surgery shows a neuropathic component. (2008) J Pain 9(10): 955-961.

25. Kinney, M.A., Hooten, W.M., Cassivi, S.D., et al. Chronic postthoracotomy pain and health-related quality of life. (2012) Ann Thorac Surg 93(4): 1242-1247.

26. Buvanendran, A., Kroin, J.S., Della Valle, C.J., et al. Perioperative oral pregabalin reduces chronic pain after total knee arthroplasty: a prospective, randomized, controlled trial. (2010) Anesth Analg 110(1): 199-207.

27. Chang, S.H., Lee, H.W., Kim, H.K., et al. An evaluation of perioperative pregabalin for prevention and attenuation of postoperative shoulder pain after laparoscopic cholecystectomy. (2009) Anesth Analg 109(4): 1284-1286.
Ommega Online Publishers

Journal Title: Journal of Anesthesia and Surgery (JAS)

Journal Short Name: J Anesth Surg
Journal ISSN: 2377-1364

E-mail: anestheisa@ommegaonline.com

Website: www.ommegaonline.org 